\title{
Blind Localization of Heating in Neural Tissues Induced by a Train of the Infrared Pulse Laser
}

\author{
Mohammad Ali Ansari*, Mahdi Zakeri \\ Optical Bio-Imaging Lab(OBI lab), Laser and Plasma Research Institute, Shahid Beheshti University, Tehran, Iran
}

*Correspondence to

Mohammad Ali Ansari, Optical

Bio-Imaging Lab(OBI lab), Laser

and Plasma Research Institute,

Shahid Beheshti University,

Tehran, Iran.

Tel: +98-21-29904022;

Email: m_ansari@sbu.ac.ir

Published online October 1 2019

\begin{abstract}
Introduction: Recently, infrared lasers (wavelengths larger than $1100 \mathrm{~nm}$ ) have been applied to stimulate neural tissues. Infrared neural stimulation (INS) has some advantages over conventional electric stimulation, including contact-free delivery, spatial precision, and lack of stimulation artifacts. In this study and based on a photothermal mechanism, we applied the heat diffusion equation to study temperature variation of a biological phantom during INS. In addition, the impact of laser parameters on spatially localized heating induced by 2 different infrared wavelengths were studied.

Methods: We studied the localization of INS inside a phantom similar to cortical neural tissue. First, we analytically solved the heat diffusion equation to study the distribution of temperature inside this phantom. Then, the accuracy of analytical results was verified by heating the phantom using amplitude-modulated infrared lasers (lambda $=1450$ and $1500 \mathrm{~nm}$, the energy between 2 and $5 \mathrm{~m}$ ) and pulse duration up to $20 \mathrm{~ms}$ ). The laser light was directed to sample by a multimode optical fiber (NA=0.22, core size $=200$ microns). Finally, the impacts of laser properties on the spatial resolution of infrared heating were discerned.

Results: In order to verify analytical results, we measured the maximum temperatures of the phantom during illumination of lasers and compared them with analytical results. The analytical results were in agreement with the experimental results. The effects of laser beam properties such as pulse duration, energy and repetition rate frequency on the spatial resolution were investigated. The results indicated that the spatial resolution of INS can be smaller than one millimeter.

Conclusion: Here, the influences of laser properties on the localization of INS inside a biological phantom were studied. These results can be applied to improve the spatial selectivity of the peripheral nerve interface.

Keywords: Infrared neural stimulation; Photothermal mechanism; Blind localized activation.
\end{abstract}

\section{Introduction}

Infrared neural stimulation (INS) is an emerging method that can be applied to directly stimulate neural systems. ${ }^{1-4}$ While the safety and efficacy of INS have been demonstrated in various studies, the typical thresholds for INS to elicit neural responses varied significant. ${ }^{5,6}$ This method provides several advantages over conventional electrical stimulation, including contact-free delivery, spatial precision, and lack of stimulation artifacts. ${ }^{7-9}$ Shapiro et al and Wells et al have demonstrated that INS can be explained by the photothermal mechanism, ${ }^{4-10}$ but the exact mechanism of INS is under discussion in the literature. ${ }^{4,7,8}$ The simulations presented by Fribance et $\mathrm{al}^{1}$ and experimental results show that a rapid increase in local temperature causes a change in membrane capacitance which generates an action potential during INS. ${ }^{4}$ This rapid increase in temperature can be modeled by the heat diffusion equation. ${ }^{5}$ Here, we analytically solved this equation - by applying an appropriate Green function - to simulate the distribution of deposited thermal energy inside a biological phantom induced by a train of the infrared pulse laser. Then these results were verified by experimental results. To do it, an aqua phantom similar to human cortical tissue was heated by 2 different wavelengths of 1450 and $1550 \mathrm{~nm}$, and then the maximal temperatures induced by infrared lasers were measured. Finally, these results were compared with analytical results. In this study, we estimated the localization of the infrared laser inside the neural phantom. The results showed that infrared laser radiations provide a possible way for localized stimulation of retinal and vestibular ganglion cells. Furthermore, they can be applied to improve the spatial selectivity of the peripheral nerve interface as motor neuron activation in peripheral nerves. ${ }^{10}$

\section{Materials and Methods}

Initially, we applied an appropriate green function to solve the heat diffusion equation analytically. Then,

Please cite this article as follows: Ansari MA, Zakeri M. Blind localization of heating in neural tissues induced by a train of the infrared pulse laser. J Lasers Med Sci. 2019;10(4):264-367. doi:10.15171/jlms.2019.43. 
we introduced the experimental set-up to measure the maximum temperature induced by an infrared laser.

Analytical Method

As mentioned above, the mechanism of INS can be explained by the photothermal mechanism. Therefore, we can apply the heat equation to determine the heat distribution during INS ${ }^{9,10}$ :

$\frac{\partial T}{\partial t}=D \nabla^{2} T(r, z, t)+\frac{1}{\rho C_{p}} S(r, z, t)(1)$

Where $T$ is temperature, $t$ is time, $r$ and $z$ are spatial coordinate transverse and longitudinal to light propagation respectively (see Figure 1). $D$ is heat conductivity, $\rho$ is tissue density, and $C_{p}$ is specific heat capacity. Herein, $S$ refers to the heat source induced by absorption of laser radiation. The absorbed energy of the laser depends on the absorption coefficient of tissue $\alpha .^{11,12}$ The laser light exiting fiber core can be described as a Gaussian divergent beam propagating through biological tissue:

$S(r, z, t)=\frac{2 \alpha E_{0}}{\pi a^{2}} \exp \left(-\frac{2 r^{2}}{a^{2}}\right) \exp (-\alpha z) f(t)$

That $a$ describes the Gaussian beam and $f(t)$ indicates the temporal shape of the laser beam. We applied an appropriate green function

$$
\begin{aligned}
& G\left(r-r^{\prime}, z-z^{\prime}, t-t^{\prime}\right)= \\
& \text { (3) } \frac{1}{\left[4 \pi\left(t-t^{\prime}\right)\right]^{\frac{3}{2}}} \exp \left(-\frac{\left(r-r^{\prime}\right)^{2}+\left(z-z^{\prime}\right)^{2}}{4 a^{2}\left(t-t^{\prime}\right)}\right)
\end{aligned}
$$

to compute $T(r, z, t)$ as the following:

$$
\begin{aligned}
& T(r, z, t)=\frac{\alpha E_{0} \exp (-\alpha z)}{4 \pi \rho C_{P} a^{2}} \\
& \int_{0}^{t} \frac{f\left(t^{\prime}\right)}{a^{2}+8 D\left(t-t^{\prime}\right)} \exp \left(\frac{-2 r^{2}}{a^{2}+8 D\left(t-t^{\prime}\right)}\right) \exp \left(\alpha^{2} D\left(t-t^{\prime}\right)\right) \operatorname{ercf}\left(\frac{2 \mathrm{D} \alpha\left(t-t^{\prime}\right)-z}{\sqrt{4 D\left(t-t^{\prime}\right)}}\right) d t^{\prime}
\end{aligned}
$$

That ercf is the complementary error function defined as $\operatorname{ercf}(\mathrm{x})=1-\operatorname{erf}(\mathrm{x})$. Eq. 4 is a general solution to the heat diffusion equation with no additional limitations or approximations and can be numerically computed. ${ }^{13} \mathrm{We}$ used this equation to estimate the maximal temperature induced by the absorption of the laser during INS.

\section{Experimental Method}

As depicted in Figure 1, the temperature of the sample induced by a pulsed laser was measured by a $\mathrm{Cu}$-Con thermocouple, and the measured data were sent to a PC by a DAQ card. In this study, we used a pulsed butterfly laser module $(\lambda=1450 \mathrm{~nm}$ and $\lambda=1550 \mathrm{~nm})$ wherein their energy and pulse duration can be adjusted up to 10 $\mathrm{mJ}$ per pulse and $20 \mathrm{~ms}$ respectively. The laser pulse was delivered to a distilled water-filled petri dish via a 200

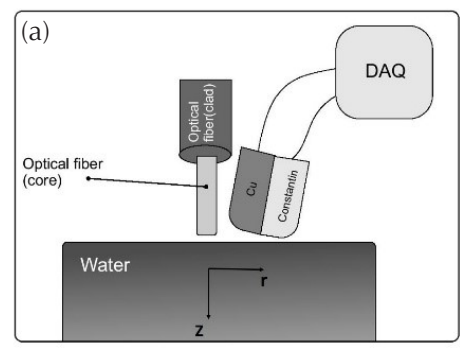

(b)

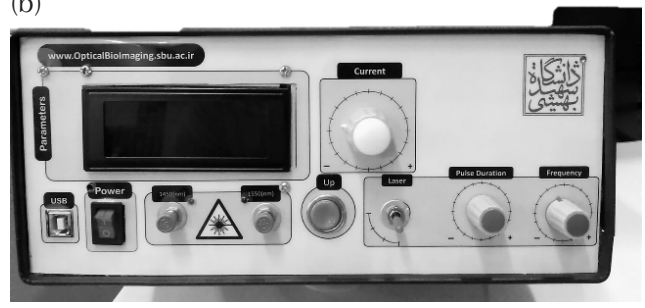

Figure 1. Schematic Set-up to Measure Maximum Temperature by $\mathrm{Cu}$ Cons Thermocouple (a). The laser light was directed to the sample by a 200 microns multimode optical fiber $(N A=0.22)$. A pulsed laser diode $(\lambda=1450 \mathrm{~nm}$ and $1550 \mathrm{~nm}$ ) with variable output energy per pulse (up to $10 \mathrm{~mJ}$ ), variable pulse width (up to $20 \mathrm{~ms}$ ) and a repetition rate (up to $10 \mathrm{~Hz}$ ) was applied to INS (b)

microns multimode optical fiber $(\mathrm{NA}=0.22)$. That was because of the similarity of the absorption coefficient of cortical nerve tissue and water for spectrum 1400-1600 $\mathrm{nm}$.

\section{Results and Discussion}

To validate the analytical method, we first estimated the maximum temperature in a homogenous phantom similar to cortical neural tissue for variable output laser energy from 2 to $5 \mathrm{~mJ}$. Then we measured the maximum temperature at depth of 100 microns with the pulse duration of $10 \mathrm{~ms}$ and the repetition rate of 2 and $5 \mathrm{~Hz}$. Several measurements showed that there was a good agreement between analytic results and measured results (for example, see Table 1). The absorption coefficient of this phantom on the wavelength of $1450 \mathrm{~nm}(1550 \mathrm{~nm})$ is approximately $3.0 \mathrm{~mm}^{-1}\left(1.0 \mathrm{~mm}^{-1}\right){ }^{11}$

The experimental results presented by Wells et $\mathrm{al}^{9}$ and $\mathrm{Bec}$ et $\mathrm{al}^{12}$ indicate that the laser radiant exposure and the repetition rate have significant effects on the rapid increase in temperature induced during INS. Here, we studied these effects by analytic and experimental methods, then the spatial temperature distribution in the neural homogenous phantom was estimated. Figure 2 depicts the effect of infrared laser parameters on spatial coordinate transverse to light illumination (in the direction of $r$, see Figure 1, and it shows the radius of the localization of the laser inside the phantom.). Figure 2(a) shows the influence of laser energy after first one second on the maximum temperature at sites laterally displaced from the center of the optical fiber $(\lambda=1450 \mathrm{~nm}, \tau=10$ $\mathrm{ms}$, and the repetition rate of $5 \mathrm{~Hz}$ ). This figure illustrates that the maximum temperature significantly decreases 
Table 1. Comparison Between Analytical Values (AV) and Experimental Data (EV) of Maximal Temperature for Different Values of Laser Energy

\begin{tabular}{lcccc}
\hline \multirow{2}{*}{ Laser Energy $(\mathbf{m}))$} & \multicolumn{2}{c}{$\mathbf{2 ~ H z}$} & \multicolumn{2}{c}{$\mathbf{5 ~ H z}$} \\
\cline { 2 - 5 } & $\mathbf{A V}\left({ }^{\circ} \mathbf{C}\right)$ & $\mathbf{E V}\left({ }^{\circ} \mathbf{C}\right)$ & $\operatorname{AV}\left({ }^{\circ} \mathbf{C}\right)$ & EV $\left({ }^{\circ} \mathbf{C}\right)$ \\
\hline 2 & 1.5 & $1.1 \pm 0.3$ & 2.2 & $4.2 \pm 0.5$ \\
3 & 2.4 & $2.5 \pm 0.7$ & 4.0 & $5.0 \pm 1.2$ \\
4 & 3.2 & $4.0 \pm 0.4$ & 5.0 & $5.2 \pm 0.1$ \\
5 & 4.0 & $5.0 \pm 0.2$ & 6.6 & $7.1 \pm 0.5$ \\
\hline
\end{tabular}

${ }^{a}$ The repetition rate frequency is 2 and $5 \mathrm{~Hz}$ and the pulse duration is $10 \mathrm{~ms}$.

for a larger value of transverse distances. The influence of the repetition rate on the variation of temperature is depicted in Figure 2(b). The intensity of radiant exposure is adjusted to be $4.4 \mathrm{~J} / \mathrm{cm}^{2}$ ( $\tau=10 \mathrm{~ms}$ ). As it is shown in Figure 2(b), an increase in the repetition rate causes an increase in temperature. It can be related to the thermal build-up that will begin to occur at a repetition rate larger than $4 \mathrm{~Hz}$. The presented results in Figure 2(b) are in agreement with those presented in Refs. ${ }^{12,14-16}$ To study the heat penetrating inside the sample, the maximal temperature at depth of 50, 100, and 200 microns were calculated (see Figure 2(c), the intensity of radiant exposure is $6.2 \mathrm{~J} / \mathrm{cm}^{2}$, the repetition rate $5 \mathrm{~Hz}$, and $\tau=$ $10 \mathrm{~ms}$ ). Figure 2(d) depicts the effect of varying pulse duration on the maximal temperature (the intensity of radiant exposure is $4.4 \mathrm{~J} / \mathrm{cm}^{2}$ ). This figure demonstrates that maximal temperature does not change with variable pulse duration. The graphs illustrated in Figure 2 reflect that most of the deposited heat is roughly localized within the radius of 400 microns. This approximated value can be controlled via laser parameters. In order to validate the localization along $\mathrm{z}$-direction (see Figure 1), the maximal temperature at 2 depths, $100 \mu \mathrm{m}$ and $200 \mu \mathrm{m}$, were measured (calculated) to be $5.0 \pm 2^{\circ} \mathrm{C}\left(5.2^{\circ} \mathrm{C}\right)$ and $2.0 \pm$ $0.1^{\circ} \mathrm{C}\left(1.8^{\circ} \mathrm{C}\right)$ respectively. Hence, most of the deposited energy can be approximately localized in a voxel size of $0.8 \times 0.8 \times 0.8 \mathrm{~mm}^{3}$.

\section{Conclusion}

Recently, INS has been applied for the stimulation of the retina nerves and the activation of the peripheral axon..$^{12,17}$ This method provides fine spatial resolution improving the selectivity of neural stimulations. In this study, we used a photothermal mechanism to estimate the spatial resolution of INS as a function of laser properties. Here, the distribution of the deposited thermal energy inside a biological phantom was estimated using the analytical and experimental methods. One can see good compatibility between the analytical and measured results. The results showed that the infrared laser can be localized in submillimeter voxel inside the biological phantom. This value of spatial resolution is obtained for conventional multimode fibers with a core size of 200 microns. These results can be applied to improve the selectivity of the peripheral nerve interface as motor neuron activation in peripheral nerves.
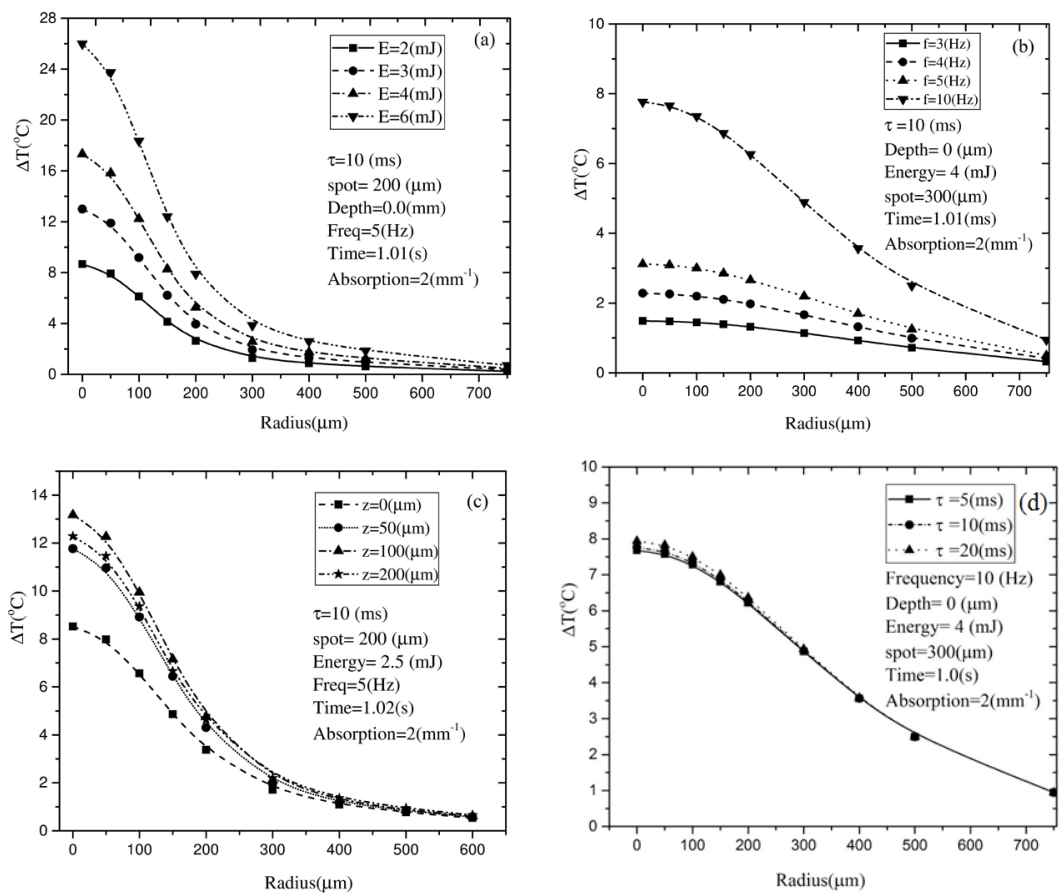

Figure 2. Calculated Maximum temperature in neural tissue induced by the infrared laser for variable laser energy (a), different repetition rates (b), at different depths, (c) and for pulse duration 5, 10 and $20 \mathrm{~ms}$ (d). 


\section{Ethical Considerations}

Not applicable.

\section{Conflict of Interests}

The authors declare no conflict of interest.

\section{Acknowledgment}

This study has been funded by a grant number D/1687/600 from Shahid Beheshti University.

\section{References}

1. Fribance S, Wang J, Roppolo JR, de Groat WC, Tai C. Axonal model for temperature stimulation. J Comput Neurosis. 2016;41(2):185-192. doi:10.1007/s10827-0160612-x.

2. Cayce JM, Friedman RM, Chen G, Jansen ED, MahadevanJansen A, Roe AW. Infrared neural stimulation of primary visual cortex in non-human primates. Neuroimage 2014;84:181-190. doi:10.1016/j.neuroimage.2013.08.040.

3. Izzo AD, Walsh JT, Jansen ED, Bendett M, Webb J, Ralph $\mathrm{H}$, et al. Optical parameter variability in laser nerve stimulation:a study of pulse duration, repetition rate, and wavelength. IEEE Trans Biomed Eng. 2007;54(6):11081114. doi:10.1109/TBME.2007.892925.

4. Shapiro MG, Homma K, Villarreal S, Richter CP, Bezanilla F. Infrared light excites cells by changing their electrical capacitance. Nat Commun. 2012;3:736. doi:10.1038/ ncomms1742.

5. Alemzadeh-Ansari MJ, Ansari MA, Zakeri M, Haghjoo $M$. Influence of radiant exposure and repetition rate in infrared neural stimulation with near- infrared lasers. $J$ Laser Med Sci. 2019. doi:10.1007/s10103-019-02741-4

6. Tan X, Rajguru S, Hunter Young NX, Stock SR, Xiao X, Richter CP. Radiant energy required for infrared neural stimulation. Sci Rep. 2015;5:13273. doi:10.1038/srep13273.

7. Norton BJ, Bowler MA, Wells JD, Keller MD. Analytical approaches for determining heat distributions and thermal criteria for infrared neural stimulation. J Biomed Opt. 2013;18(9):098001. doi:10.1117/1.JBO.18.9.098001.

8. Liljemalm R, Nyberg $T$, von Holst $H$. Heating during infrared neural stimulation. Lasers Surg Med. 2013;45(7):469-481. doi:10.1002/lsm.22158.

9. Wells J, Kao C, Konrad P, Milner T, Kim J, MahadevanJansen A, et al. Biophysical mechanisms of transient optical stimulation of peripheral nerve. Biophys $J$. 2007;93(7):2567-2580. doi:10.1529/biophysj.107.104786.

10. Peterson EJ, Tyler DJ. Motor neuron activation in peripheral nerves using infrared neural stimulation. J Neural Eng. 2013;11(1):016001. doi:10.1088/1741-2560/11/1/016001.

11. Ansari MA, Erfanzadeh M, Mohajerani E. Mechanisms of laser-tissue interaction: II. Tissue thermal properties. $J$ Lasers Med Sci. 2013;4(3):99-106.

12. Bec JM, Albert ES, Marc I, Desmadryl G, Travo C, Muller A, et al. Characteristics of laser stimulation by near infrared pulses of retinal and vestibular primary neurons. Lasers Surg Med. 2012;44(9):736-45. doi:10.1002/lsm.22078.

13. Ansari MA, Mohajerani, E. Mechanisms of laser-tissue interaction: optical properties of tissue. J Lasers Med Sci. 2011;2(3):119-125.

14. Welch AJ, Van Gemert MJ, Optical-Thermal Response of Laser-Irradiated Tissue. Berlin: Springer; 2011.

15. Thompson AC, Wade SA, Brown WG, Stoddart PR. Modeling of light absorption in tissue during infrared neural stimulation. J Biomed Opt. 2012;17(7):0750021-6. doi:10.1117/1.JBO.17.7.075002.

16. Thompson AC, Wade SA, Cadusch PJ, Brown WG, Stoddart PR. Modeling of the temporal effects of heating during infrared neural stimulation. $J$ Biomed Opt. 2013;18(3):035004. doi:10.1117/1.JBO.18.3.035004.

17. You M, Mou Z. Model study of combined electrical and near-infrared neural stimulation on the bullfrog sciatic nerve. Lasers Med Sci. 2017;32(5):1163-1172. doi:10.1007/ s10103-017-2222-x 